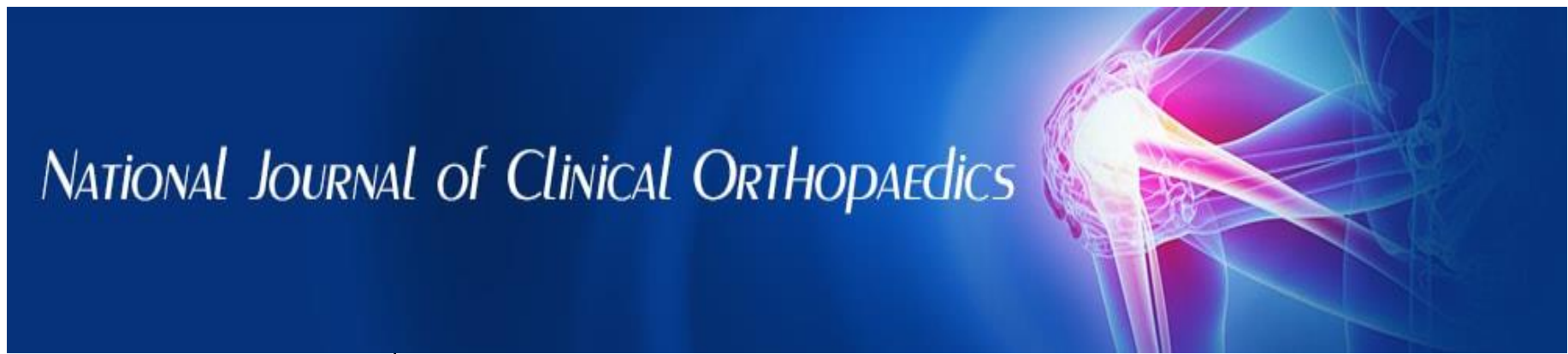

ISSN (P): 2521-3466

ISSN (E): 2521-3474

(C) Clinical Orthopaedics www.orthoresearchjournal.com 2021; 5(4): 75-78

Received: 01-08-2021

Accepted: 03-09-2021

Wanlamkupar Khongwir Department of Orthopaedics \& Trauma, The North Eastern Indira Gandhi Regional Institute of Health \& Medical Sciences,

Shillong, Meghalaya, India

Dr. Tashi G Khonglah Department of Orthopaedics \& Trauma, The North Eastern Indira Gandhi Regional Institute of Health \& Medical Sciences, Shillong, Meghalaya, India
Corresponding Author: Dr. Tashi G Khonglah Department of Orthopaedics \& Trauma, The North Eastern Indira Gandhi Regional Institute of Health \& Medical Sciences, Shillong, Meghalaya, India

\section{Functional outcome following internal fixation of rare type IV capitellum fractures using varied fixation methods: A case series}

\author{
Wanlamkupar Khongwir and Dr. Tashi G Khonglah
}

DOI: https://doi.org/10.33545/orthor.2021.v5.i4b.329

\section{Abstract}

Background: Capitellum fractures are very rare injuries, accounting for less than $1 \%$ of elbow fractures. These fractures are difficult to interpret on a plain radiograph and usually require a computed tomography scan to understand the complexity of the fracture. Treatment of such fractures vary from conservative treatment in the form of closed reduction and immobilization, excision of the fragment to open reduction and internal fixation with $\mathrm{K}$ wires, partially threaded $4 \mathrm{~mm}$ cancellous screws or Herbert screws. In this paper we present the outcome of four cases of Type IV capitellum fractures that were managed operatively by different methods.

Materials \& Methods: Four patients were treated in our hospital from 2018 to 2019, for Type IV capitellum fractures. The mechanism of injury was a fall on the out stretched hand in three patients while the fourth patient was involved in a motor cycle accident. The fractures were equally distributed on either elbows, two on each side. The three males patients were aged between 18 to 30 years while the lone female was 40 years of age. All patients were operated in the supine position under general anaesthesia, using a tourniquet and with fluoroscopic guidance. Surgery was performed through an extended lateral Kocher's approach. Definitive fixation in two patients was by using $2.7 \mathrm{~mm}$ Herbert screws alone while in one with a lateral condyle humerus fracture, fixation was supplemented using plate and screws. K-wire fixation was used for one patient.

Results: 2 patient attained a score of excellent in the Mayo Elbow Performance Score evaluation while 1 patient each had a good and a fair score. There was no evidence of arthritis, avascular necrosis or heterotopic ossification till 1 year of follow-up.

Conclusion: Flawless reduction and fixation of the fragment with appropriate postoperative rehabilitation protocol are crucial to achieving optimal results.

Keywords: capitellum fracture, internal fixation, Herbert screws, k-wires, mayo elbow performance score

\section{Introduction}

Capitellum fractures are very rare injuries, accounting for less than $1 \%$ of all elbow fractures ${ }^{[1-}$ 2]. These fractures are challenging to interpret on plain radiographs and often require computed tomography scans to comprehend their complexity. Typically, such injuries occur following a low energy fall on the outstretched hand or by direct axial compression, with the elbow in a semi flexed position.

Hahn in 1853, described an isolated capitellum fracture [3] whereafter in 1898, Steinthal described an identical injury ${ }^{[4]}$. The Hahn-Steinthal or the Type I fracture involves the capitellum articular surface along with the subchondral bone ${ }^{[5]}$. The Type II fracture (KocherLorenz fracture) is a cartilaginous articular fracture of the capitellum and may include a small fragment of sub-chondral bone typically described as "uncapping" of the capitellum ${ }^{[6]}$. Bryan and Morrey later modified the classification with a Type III to incorporate comminuted capitellum fractures. The Type IV capitellum fractures was later added by McKee et al. ${ }^{\text {[7], }}$ which comprised of Type I fracture with the extension to the lateral half of the trochlea. According to AO classification, capitellum fractures has been classified as B3.1, trochlea fractures as B3.2 and if it involved both, then B3.3 ${ }^{[11]}$. Dubberley classified capitellum fractures into three types that correlated to clinical outcome. Type 1, with involvement primarily of the capitellum with or without lateral trochlea ridge. 
Type 2 fractures with involvement of both capitellum and trochlea, and Type 3 fractures of both capitellum and trochlea in separate fragments. These authors noted that fracture complexity was commensurate to worsened outcomes ${ }^{[12]}$.

Treatment of capitellum fractures vary from conservative treatment in the form of closed reduction and immobilization ${ }^{[8,}$ 9], excision of the fragment to open reduction and internal fixation with $\mathrm{K}$ wires ${ }^{[2,8]}$, partially threaded $4 \mathrm{~mm}$ cancellous screws or Herbert screws ${ }^{[10]}$.

The purpose of this study was to evaluate the outcome following surgical management of type IV capitellum fractures in four patients that were managed through different methods.

\section{Material and Methods}

Four patients, three males and a female were treated in our hospital from 2018 to 2019, for type IV capitellum fractures. In three patients, the mechanism of injury was a fall on the out stretched hand while the fourth patient was involved in a motor cycle accident. The fractures were equally distributed on either elbows, two on each side. The three males patients were aged between 18 to 30 years while the lone female was 40 years of age.

Anteroposterior and lateral view radiographs of the elbows were taken and revealed the pathognomic double arc sign. Computed tomograms of all the cases exhibited Mc Kee Type IV fractures of the capitellum with one involving the lateral condyle of the humerus.

All patients were operated in the supine position under general anaesthesia, using a tourniquet and with fluoroscopic guidance. Following anaesthesia, the elbow was assessed clinically for ligamentous instability. Through an extended lateral Kocher's approach, the incision was made from the anterior aspect of the lateral column of the distal humerus to approximately $2 \mathrm{~cm}$ distal to the radial head. The common extensor origin was raised sub periosteally as a flap along with the anterior capsule. The exposure was then extended distally between the Anconeus and the Extensor Carpi Ulnaris. The origin of the lateral collateral ligamentous complex was not disturbed. The forearm was kept pronated all throughout the surgery with special attention to avoid injury to the posterior interosseous nerve. A bone lever was kept over the medial column which aided visualization of the entire fracture area. Copious amounts of Normal Saline was used to irrigate and to clear the joint cavity of any debris. The fracture was reduced; assessed clinically and temporarily held in place using $1.5 \mathrm{~mm} \mathrm{~K}$-wires. Fracture reduction was confirmed using an image intensifier in both the anteroposterior and lateral views. Definitive fixation in two patients was by using $2.7 \mathrm{~mm}$ Herbert screws (Figure 1) alone while in one with a lateral condyle humerus fracture, fixation was supplemented using plate and screws (Figure 2). K-wire fixation was used in one patient, the direction was from posterior to anterior through the posterior aspect of the lateral condyle (Figure 3). The joint was irrigated thoroughly with normal saline and wound closed in layers following adequate haemostasis.

Post-operative, all cases were immobilized with an above elbow Plaster of Paris slab with the elbow kept at 90 degrees flexion and the forearm in neutral rotation. Wound inspection was done 2 days after the surgery and stitches were removed on the 14 days. In three patients whose fractures were fixed using Herbert screws, mobilisation exercises were initiated immediately after removal of stitches. In the lone patient in whom the fracture was fixed using $\mathrm{K}$-wires, mobilisation was delayed at 4 weeks after the surgery. All patients were followed up weekly during the first one month and thereafter every month for a year. During the follow up, repeat radiographs were taken. Range of motion, and MEPS (Mayo Elbow Performance Score) was assessed during each visit for every patient by the operating surgeon.

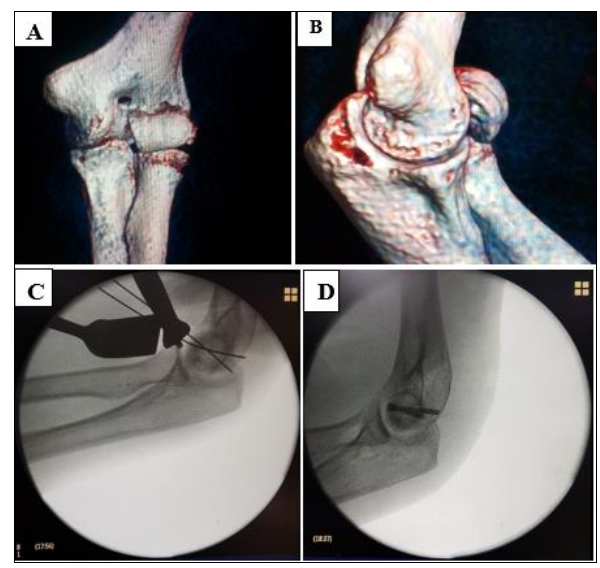

Fig 1: (A \& B) - 3D CT scan showing capitellum fracture involving part of the trochlea. (C) Intraoperative fracture reduction (D) Fracture fixed using two Herbert screws

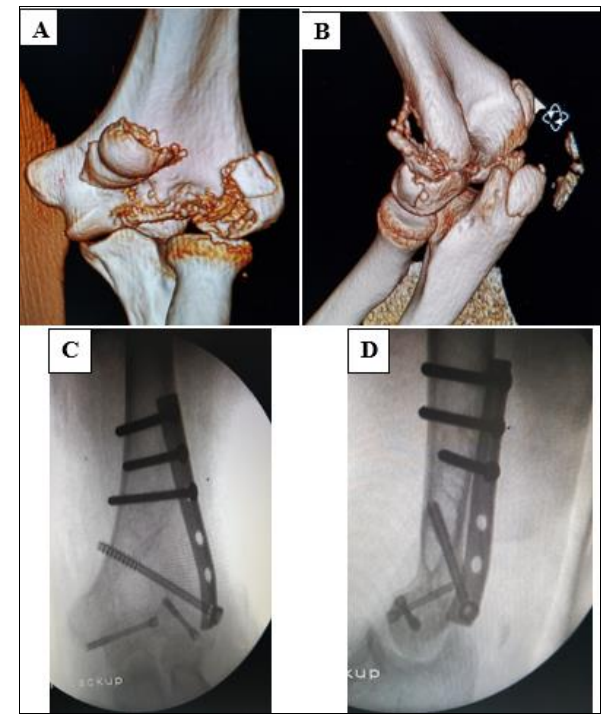

Fig 2: (A \& B) 3D CT Scan showing a Type IV capitellum fracture with a communited lateral condyle fracture. $(C \& D)$ Final fracture fixation as seen in anteroposterior and lateral views
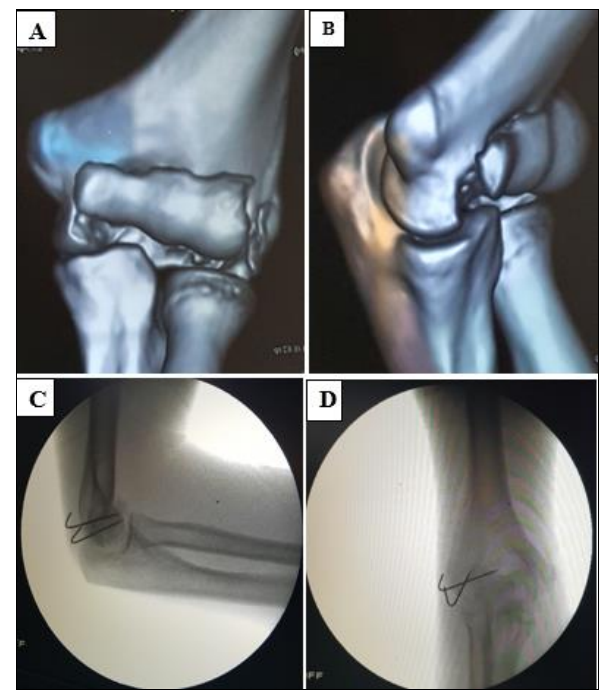

Fig 3: (A \& B) 3D CT Scan showing capitellum fracture and most part of the trochlea. $(C \& D)$ Fluoroscopy image showing fracture fixed by two $\mathrm{K}$ wires 


\section{Results}

The mean age of the patients ( 3 males and a female) who had undergone surgery was 27 years (range 18 - 40 years). The mechanism of injury was a fall on the outstretched hand for the male patients; however the female patient had a road traffic accident. The female patient had an associated lateral condyle fracture of the elbow. Though all the patients attended emergency on the day of trauma, the lone female patient initially opted for traditional massage for three weeks. All patients were operated within three days of admission. There were no intraoperative complications and no distal neurovascular deficit.
All the patients were assessed clinically and radiologically during their follow-up. The mean flexion was 131.25 degrees (range 120-140 degrees) and mean extension was 2.5 degrees (range 0-5degrees). The mean pronation-supination were 78.75 degrees (range 70- 85 degrees) and 88.75 degrees (range 85-90 degrees) respectively. 2 patient attained an excellent score during the Mayo Elbow Performance Score evaluation, 1 patient attained a good score while the female patient with associated lateral condyle fracture had a fair result (Table 1). There was no evidence of arthritis, avascular necrosis or heterotopic ossification till 1 year of follow-up.

Table 1: Functional outcome of patients following surgery at 12 months follow-up

\begin{tabular}{|c|c|c|c|c|c|c|c|}
\hline S. No. & Age (years) & Sex & Laterality & Implants used & ROM extension-flexion & ROM pronation-supination & MEPS \\
\hline 1 & 18 & Male & Right & K-wires & $5-130$ & Good \\
\hline 2 & 21 & Male & Left & Herbert screws & $5-135$ & $85-85$ \\
\hline 3 & 29 & Male & Right & Herbert screws & $0-140$ & $80-90$ \\
\hline 4 & 40 & Female & Left & Herbert screws, plate and screws & $0-120$ & Excellent \\
\hline
\end{tabular}

Note: ROM- Range of motion in degrees, MEPS - Mayo Elbow Performance Score

\section{Discussion}

Capitellum fractures are more common in females than in males with ratio $4: 1$. It is rarely seen in children with most cases occurring above 12 years of age ${ }^{[2]}$. In children radiological diagnosis is difficult because the capitellum is not fully ossified before age 10 years and an oblique radiograph has been suggested to detect the fracture ${ }^{[13]}$. In adults, capitellum fractures particularly type IV are very rare and difficult to diagnose on regular radiographic views of the elbow. On the lateral view of the elbow, the "double arc sign" has been reported to be pathognomic of these fractures because of the lateral part of the trochlea and capitellum superimpose on each other ${ }^{[5]}$. Majority of these are complex intra-articular fractures and a 3D computer tomography scan is a requisite to understand the geometry of fracture and to also aid in proper preoperative planning.

Management of capitellum fractures vary from conservative, to fixation and to excision of the fragments. In 1996, Ochner et al., successfully reported nine cases of coronal capitellum fractures treated by closed reduction with long term follow up ${ }^{[8]}$. In our series, no attempt of closed reduction was made, considering the possibility of non-anatomical reduction, arthritis and loss of motion of the elbow ${ }^{[14]}$. Excision of the capitellum can lead to instability of the elbow. Fragment excision by some authors is suggested to prevent avascular necrosis and radio-humeral osteoarthritis due to displaced fragment. Avascular necrosis is uncommon after surgical management, however feared as a major complication. These fractures, despite of being void of soft tissue attachments unite because of rapid revascularisation of the of the fracture fragment ${ }^{[15]}$. In our series, all patients had a pain free elbow which was stable and with no sign of avascular necrosis. A free capitellar fragment should not discourage a surgeon to opt for internal fixation, due to low incidence of avascular necrosis ${ }^{[12,15]}$.

We used the extended lateral Kocher's approach for fixation of all these complex fractures. Other approach like the posterior approach with olecranon osteotomy has been described by Sano et al. for proper visualisation of the trochlea ${ }^{[16]}$. In our series we did not find necessary, and by proper retraction of the medial structures the trochlea were clearly visible. The fracture fragments after anatomical reduction were temporally fixed with $\mathrm{K}$-wires and confirmed under fluoroscopy. Small, loose bony fragments should be discarded and the joint must be thoroughly irrigated. In the literature many fixation device has been mentioned including K-wires [1, 17], 4mm cancellous screw [17], Herbert screws ${ }^{[1,7,17]}$ and absorbable polyglycolide pins ${ }^{18}$. We used Herbert screws in two case, K-wires in one and Herbert screws with plate supplement in another (associated with lateral condyle humerus fracture). Poynton et al. reported that Kirschner wires do not provide enough stability for mobilisation before fracture healing and damaged the articular surface. However, in our case with delayed mobilisation of four weeks and gradual exercise we achieved a good result. Removal of Kirschner wire was done at six weeks after the fracture has consolidated.

Complications like elbow stiffness and pain are the most common with forearm rotation rarely affected ${ }^{[19,20]}$. The major factors for the loss of elbow range of motion were articular damage and deformity, extra-articular or intra-articular adhesions and improper postoperative mobilisation protocol [21]. Avoiding these factors are keys to achieve good functional results.

\section{Conclusion}

Type IV capitellum fractures are very rare injuries. A thorough clinical examination with good radio imaging are paramount for verifying the diagnosis. Various fixation methods and implants have been described in literature. However, the accurate reduction and adequate fixation of the fragment with proper postoperative rehabilitation protocol are keys to achieving optimum functional results.

Due to the rare nature of this injury, only a small number of patients could be included in this study. The duration of followup also was another limitation whereby we were unable to comprehend other late complications associated with this fracture. A larger number of patients with an increased duration of follow-up would definitely help us to understand better the overall outcome of this fracture.

\section{References}

1. Poynton AR, Kelly IP, O'Rourke SK. Fractures of the capitellum: A comparison of two fixation methods. Injury 1998;29:341-3.

2. Alvarez E, Patel MR, Nimberg G, Pearlman HS. Fractures of the capitulum humeri. J Bone Joint Surg Am 1975;57:1093-6.

3. Hahn NF. Fall von einer besonderes varietat der frakturen des ellenbogens. Zeitsch-Wundartze Geburtshlefer 
$1853 ; 6: 185$.

4. Steinthal D. Die isolirte fraktur der eminenthia capetala in ellenbogengelenk. Zentralb Chir 1898;15:17.

5. Bryan RS, Morrey BF. Fractures of the distal humerus. In: Morrey BF, editor. The Elbow and Its Disorders. Philadelphia: WB Saunders 1985, 302-339.

6. McKee MD, Jupiter JB, Bamberger HB. Coronal shear fractures of the distal end of the humerus. J Bone Joint Surg Am 1996;78:49-54.

7. McKee MD, Jupiter JB, Bamberger HB. Coronal shear fractures of the distal end of the humerus. J Bone Joint Surg Am 1996;78(1):49-54.

8. Ochner RS, Bloom H, Palumbo RC, Coyle MP. Closed reduction of coronal fractures of the capitellum. J Trauma 1996;40:199-203.

9. Mazel MS. Fracture of the capitellum: A report of a case. $\mathbf{J}$ Bone Joint Surg Am 1935; 17:483-8.

10. Lambert SM, Pike J, Railton GT. Fractures of the humeral capitellum: Herbert screw fixation. J R Coll Surg Edinb 1994;39:321-3.

11. Marsh JL, Slongo TF, Agel J et al. Fracture and dislocation classification compendium, 2007: Orthopaedic Trauma Association classification, database and outcomes committee. J Orthop Trauma 2007;21(10):S1-133.

12. Singh AP, Singh AP, Vaishya R et al. Fractures of capitellum: a review of 14 cases treated by open reduction and internal fixation with Herbert screws. Int Orthop 2010;34(6):897-901.

13. Pradhan BB, Bhasin D, Krom W. Capitellar fracture in a child: The value of an oblique radiograph: A case report. J Bone Joint Surg Am 2005;87:635-8.

14. Schindler OS. Bilateral capitellum humeri fracture: A case report and review of the literature. J Orthop Surg (Hong Kong) 2003;24(11):207-12.

15. Mehdian H, McKee MD. Fractures of capitellum and trochlea. Orthop Clin North Am 2000;31(1):115-27.

16. Sano S, Rokkaku T, Saito S, Tokunaga S, Abe Y, Moriya H. Herbert screw fixation of capitellar fractures. J Shoulder Elbow Surg 2005;14:307-11.

17. Letts $M$, Rumball $\mathrm{K}$, Bauermeister S, McIntyre W, D'Astous J. Fractures of the capitellum in adolescents. J Pediatr Orthop 1997; 17:315-20.

18. Bilić R, Kolundzić R, Anticević D. Absorbable implants in surgical correction of a capitellar malunion in an 11-year old: A case report. J Orthop Trauma 2006;20:66-9.

19. Ruchelsman DE, Tejwani NC, Kwon YW et al. Coronal plane partial articular fractures of the distal humerus: current concepts in management. J Am. Acad. Orthop Surg 2008;16(12):716-28.

20. Ring D. Open reduction and internal fixation of an apparent capitellar fracture using an extended lateral exposure. J Hand Surg Am 2009;34(4):739-44.

21. Mehdian H, McKee MD. Fractures of capitellum and trochlea. Orthop Clin North Am 2000;31(1):115-27. 